Abstract P09 Table 1

\begin{tabular}{lll}
\hline & $\begin{array}{l}\text { Training cohort } \\
\text { AUR0C }(\mathbf{9 5 \%} \text { CI) }\end{array}$ & $\begin{array}{l}\text { Validation cohort } \\
\text { AUROC }(\mathbf{9 5 \%} \text { CI) }\end{array}$ \\
\hline NAS 0-2 vs NAS 3-8 & $0.833(0.700$ to 0.966$)$ & $0.829(0.737$ to 0.921$)$ \\
NAS 0-3 vs NAS 4-8 & $0.854(0.763$ to 0.945$)$ & $0.784(0.668$ to 0.900$)$ \\
NAS 0-4 vs NAS 5-8 & $0.865(0.772$ to 0.957$)$ & $0.723(0.505$ to 0.941$)$ \\
\hline
\end{tabular}

for ascertaining prognosis and the stratification of patients for existing interventions and emerging therapies. Liver biopsy is considered the reference standard for assessing NASH. The aim of this study was to develop a biomarker of NASH which would be able to detect NASH prior to the development of significant hepatic fibrosis. Candidate biomarkers of hepatic inflammation, apoptosis and liver fibrosis were selected on the basis of biological plausibility and previous association with NAFLD.

Method 172 patients with NAFLD and no evidence of other liver disease were consecutively recruited from two centres. 84 patients from the first centre were included as a training cohort and 88 patients from the second centre as a validation cohort. Liver biopsies were performed on all patients and scored using the NAFLD activity score (NAS). 36 patients with advanced fibrosis were excluded from the analysis. Serum samples were taken on all patients and tested for five matrix proteins (HA, P3NP, TIMP-1, Procollagen 4 and YKL-40) and 19 haematological and biochemical parameters including HOMAIR. Stepwise multiple linear regression was used to determine the relationship of the multiple variables to the NAS score.

Results A model combining terminal peptide of pro-collagen III (P3NP) and tissue inhibitor of matrix metalloproteinase 1 (TIMP-1) was found to significantly correlate with the NAS score in the training cohort $(\mathrm{R}=0.643, \mathrm{R} 2=0.413, \mathrm{p}<0.0000001)$. The regression model was then validated in the second patient cohort. AUROC were calculated for the ability of the model to discriminate between differing degrees of NAS.

Conclusion This model appears to have good diagnostic performance for the detection of NASH in both the training and validation cohorts. Our results appear promising and if confirmed in further studies this model will be of clinical utility in detecting the minority of patients with NAFLD who have NASH and are at risk of developing progressive liver disease.

\section{P10 WHOLE-EXOME-SEQUENCING-BASED DISCOVERY OF NOVEL SYNDROMIC FORM OF NEONATAL CHOLESTASIS}

doi:10.1136/gutjnl-2011-300857a.10

${ }^{1} \mathrm{~J}$ L Hartley, ${ }^{2} \mathrm{M}$ Simpson, ${ }^{3} \mathrm{~L}$ Tee, ${ }^{4} \mathrm{R}$ M Brown, ${ }^{5} \mathrm{~S}$ Kirkham, ${ }^{3} \mathrm{~S}$ Pasha, ${ }^{2} \mathrm{R}$ C Trembath, ${ }^{3} \mathrm{P}$ Gissen, ${ }^{3} \mathrm{E}$ R Maher, ${ }^{1} \mathrm{D}$ A Kelly, ${ }^{3} \mathrm{~N}$ V Morgan. ${ }^{1}$ Birmingham Children's Hospital; ${ }^{2}$ King's College Hospital, London; ${ }^{3}$ Birmingham University; ${ }^{4}$ University Hospitals Birmingham; ${ }^{5}$ Oueen's Medical Centre, Nottingham

Introduction Two cousins from a consanguineous family presented with low $\gamma$ glutamyl transferase (GGT) cholestasis, trichorrhexis nodosa (TN) and severe hypoglycaemia which required diazoxide to stabilise. One child also had life threatening diarrhoea necessitating parenteral nutrition, which suggested the possible diagnosis of trichohepatoenteric syndrome (THES). However screening of the THES gene (TTC37) excluded mutations.

Aim The aim of this study was to identify the molecular genetic defect in this family and hence further understanding of unexplained cholestasis within a multisystem disorder.

Method We used a novel combination of autozygosity mapping combined with whole-exome-sequencing (WES). An Affymetrix
250K SNP chip genome-wide linkage scan was used to identify common regions of shared homozygosity. SureSelect human All Exon kit (Agilent Technologies) and Illumina GaIIx was used for WES of both individuals. Single nucleotide substitutions and small insertion deletions were identified. Filtering of variants for novelty was performed by comparison to dbSNP131 and 1000 Genomes pilot SNP calls (March 2010) and variants identified in 40 control exomes sequenced and analysed by the same method described above.

Results The largest overlapping autozygous regions were at chromosome 7, 16, 20, 12 and 4. The whole exome sequencing identified 17844 and 17867 variations in patients 1 and 2 respectively. Of these only three homozygous non-synonymous variants and one frameshift variant were found in both patients in the identified homozygous regions. The frameshift was a homozygous single base $\mathrm{G}$ deletion (c.587delG) in exon 6 of $A K R 1 D 1$ which mapped within the candidate homozygous region in chromosome 7 . The variant results in a frameshift at amino acid 196 leading to a premature stop codon 11 amino acids downstream (p. Cys196SerfsX11).AKR1D1 encodes the enzyme $\delta^{4}$-3-oxosteroid $5 \beta$ reductase that is required for the synthesis of chenodeoxycholic and cholic acids important for normal bile flow. Mutations in $A K R 1 D 1$ have previously been described in patients with severe neonatal liver disease.

Conclusion In conclusion we have extended the clinical features of bile salt synthesis disorders resulting from mutations in $A K R 1 D 1$ to include a severe form of low GGT cholestasis, TN and severe hypoglycaemia which may be amenable to treatment with bile salt supplementation. Combining the technique of whole genome linkage mapping and WES creates a powerful tool to elucidate the molecular basis of uncharacterised genetic disorders.

\section{P11 INDUCTION OF HYPERAMMONAEMIA FROM THE SMALL AND LARGE INTESTINE IN PATIENTS WITH CIRRHOSIS WITH MAGNETIC RESONANCE OUANTIFICATION OF BRAIN WATER AND METABOLITES}

doi:10.1136/gutjnl-2011-300857a.11

${ }^{1} \mathrm{H}$ Mardini, ${ }^{1} \mathrm{~F}$ Smith, ${ }^{2} \mathrm{~A}$ Blamirel, ${ }^{2} \mathrm{C}$ Record. ${ }^{1}$ Royal Victoria Infirmary, Newcastle Upon Tyne and Institute of Cellular Medicine, Newcastle University; ${ }^{2}$ Newcastle Magnetic Resonance Centre, Newcastle University, UK

Introduction Induction of hyperammonaemia from glutaminase action in the small intestine ${ }^{1}$ or systemic catabolism of oral amino acids $^{2}{ }^{3}$ is well recognised but, although suggested in 1959 by Walser and Bodenlos, ${ }^{4}$ ureolysis resulting in hyperammonaemia has not been demonstrated from urea delivered to the colon. Hepatic encephalopathy (HE) is now thought to be caused by cerebral oedema. Aim We hypothesised that if hyperammonia is a key factor in hepatic encephalopathy, induced hyperammonaemia from oral amino acid or urea challenge would lead to transient changes in brain water distribution and metabolite concentration.

Method Amino acid (mixture of equal parts of glycine, serine and threonine) or urea challenges were undertaken in 18 patients with stable cirrhosis 5 of whom gave a history of hepatic encephalopathy. Sequentail blood ammonia concentration was determined with the ammonia checker and brain water and metabolites with magnetic resonance diffusion tensor imaging and spectroscopy.

Results Oral urea and amino acids (delivered to the colon by coating) resulted in peak increments in blood ammonia of $146 \pm$ SD 164 and $55 \pm 51 \mu \mathrm{mol} / \mathrm{l}$ while for uncoated amino acids the mean increment was $58( \pm 41) \mu \mathrm{mol} / \mathrm{l}$. The latter was accompanied by a significant increase in $A D C$ of $9 \%(p=0.004)$. Increased ADC was significantly correlated with blood ammonia $(r=0.58, p=0.04)$. The change in ammonia levels also correlated with the increase in 
glutamine levels $(\mathrm{r}=0.78, \mathrm{p}=0.002)$. Myo-inositol concentration decreased significantly by $0.7( \pm 0.7) \mathrm{mMol} / 1$ between scans and this correlated with the mean difference in $\mathrm{ADC}(\mathrm{r}=0.59, \mathrm{p}<0.04)$.

Conclusion These results show that hyperammonia can be derived from nitrogenous substrates in the colon and can directly drive changes in brain water distribution as a mechanism for cerebral oedema development. Since cerebral astrocytes contain glutamine synthetase, our MRS data suggests intracerebral formation of glutamine from ammonia. Developments in therapy for hepatic encephalopathy need to focus on amelioration of colonic ammonia formation.

\section{REFERENCES}

Oppong K, Al Mardini H, Thick M, et al. Hepatology 1997;26:870-6.

Douglass A, Al Mardini H, Record C. J Hepatol 2001;34:658-64.

Al Mardini H, Douglass A, Record C. Metab Brain Dis 2006;21:1-10.

Walser, Badenlos. J Clin Invest 1959;38:1617-26.

\section{P12 MEASURING THE EFFECTIVENESS OF A MULTIDISCIPLINARY NASH CLINIC}

doi:10.1136/gutjnl-2011-300857a.12

${ }^{1} \mathrm{C}$ M Peake, ${ }^{2} \mathrm{~J} \mathrm{~F} \mathrm{~L} \mathrm{Cobbold,}{ }^{3} \mathrm{O} \mathrm{M}$ Anstee, ${ }^{4} \mathrm{M}$ Yee, ${ }^{2} \mathrm{M}$ R Thursz. ${ }^{1}$ Imperial College, London, UK; ${ }^{2}$ Hepatology and Gastroenterolgy Section, Department of Medicine, Imperial College, London, UK; ${ }^{3}$ Institute of Cellular Medicine, Newcastle University, Newcastle-Upon-Tyne, UK; ${ }^{4}$ Division of Diabetes, Endocrinology and Metabolism, Department of Medicine, Imperial College, London, UK

Introduction Non-alcoholic fatty liver disease (NAFLD) and specifically non-alcoholic steatohepatitis (NASH) are associated with both increased liver-related and cardiovascular morbidity and mortality. A multi-disciplinary, individualised approach targeting the complex pathogenesis of the disease has been employed in a tertiary/secondary care setting. The aims of this study were (1) to investigate the effectiveness of a multi-disciplinary NASH clinic by assessing the change in liver disease markers and risk factors for liver-related and cardiovascular mortality over time, and (2) to identify factors that influence patient response to treatment.

Method This was a retrospective survey of current clinical practice. NASH/NAFLD was defined histologically or NAFLD by echogenic liver on ultrasound with raised aminotransferase values in absence of a significant alcohol history or other hepatic co-morbidities. Interventions included: lifestyle advice; dietetic input; exercise classes; pharmacological therapy; bariatric surgery. Clinical and anthropometric data were collected including serum ALT, BMI, HBA1c, systolic blood pressure, total and HDL cholesterol values and analysed for the cohort overall and for patients who were obese, diabetic, hypertensive and dyslipidaemic respectively. Responders to treatment were defined as those with $>10 \%$ decrease in ALT over the study period. Univariate and multivariate analysis were conducted to analyse baseline factors influencing patient response.

Results 145 patients were included with median follow-up of 12.5 months (range 3-44 months). Overall improvement was seen in ALT $\left(-15 \%, \mathrm{p}=7 \times 10^{-6}\right)$, BMI $\left(-1.5 \%, \mathrm{p}=6 \times 10^{-6}\right)$ and total cholesterol $(-4.1 \%, p=0.006)$. BMI improved by $>10 \%$ in $8 \%$, by $>7 \%$ in $16 \%$ and by $>5 \%$ in $23 \%$ of patients. Patients categorised by abnormal baseline ALT, baseline obesity, baseline hypertension and baseline dyslipidaemia had improvements in ALT $(-19 \%$, $\left.\mathrm{p}=1 \times 10^{-7}\right)$, BMI $(-2.4 \%, \quad \mathrm{p}=0.001)$, systolic BP $(-5.4 \%$, $\left.\mathrm{p}=3 \times 10^{-4}\right)$ and total cholesterol $(-5.5 \%, \mathrm{p}=0.002)$ respectively. Patients with type 2 diabetes made up a higher proportion of those who did not respond or who progressed compared to those who responded on univariate analysis $(p=0.02)$, but this was not significant on multivariate analysis. Moreover, patients with diabetes did not have a significant decrease in ALT $(-8 \%, p=0.06)$.

Conclusion The management framework adopted by the multidisciplinary NASH clinic is effective at reducing ALT overall Cardiovascular risk factors were improved overall. Diabetic patients had a poor ALT response. These data support the use of a multidisciplinary NASH clinic, but long-term outcome data are awaited.

\section{P13 THE PERFORMANCE VALIDITY OF BREATH SAMPLE ANALYSIS IN THE DIAGNOSIS OF HEPATIC ENCEPHALOPATHY IN PATIENTS WITH CIRRHOSIS}

doi:10.1136/gutjnl-2011-300857a.13

${ }^{1}$ E Halliday, ${ }^{2} S$ Stevens, ${ }^{1} \mathrm{M}$ Stubbs, ${ }^{3} \mathrm{R}$ Morris, ${ }^{1} \mathrm{M}$ Morgan. ${ }^{1}$ Centre for Hepatology, University College London Medical School, London, UK; ${ }^{2}$ Centre for Research in Analytical, Materials and Sensor Science, University of the West of England, Bristol, UK: ${ }^{3}$ Research Department of Primary Care and Population Health, University College London, London, UK

Introduction Hepatic encephalopathy (HE) has a detrimental effect on patients' health-related quality of life and a significant negative impact on survival. Nevertheless, there is no diagnostic gold standard so the condition is often poorly diagnosed and managed.

Aim The aim of this study was to evaluate the performance validity of breath sample analysis for volatile organic compounds (VOCs) in the diagnosis of hepatic encephalopathy (HE) in patients with cirrhosis.

Method The patient population comprised 26 individuals (17 men, 9 women) of mean (range) age 60 (45 to 75) years, with biopsy-proven cirrhosis secondary to alcohol $(n=21)$, or non-alcoholic steatosis, hepatitis C, autoimmune hepatitis, haemochromatosis or cryptogenic

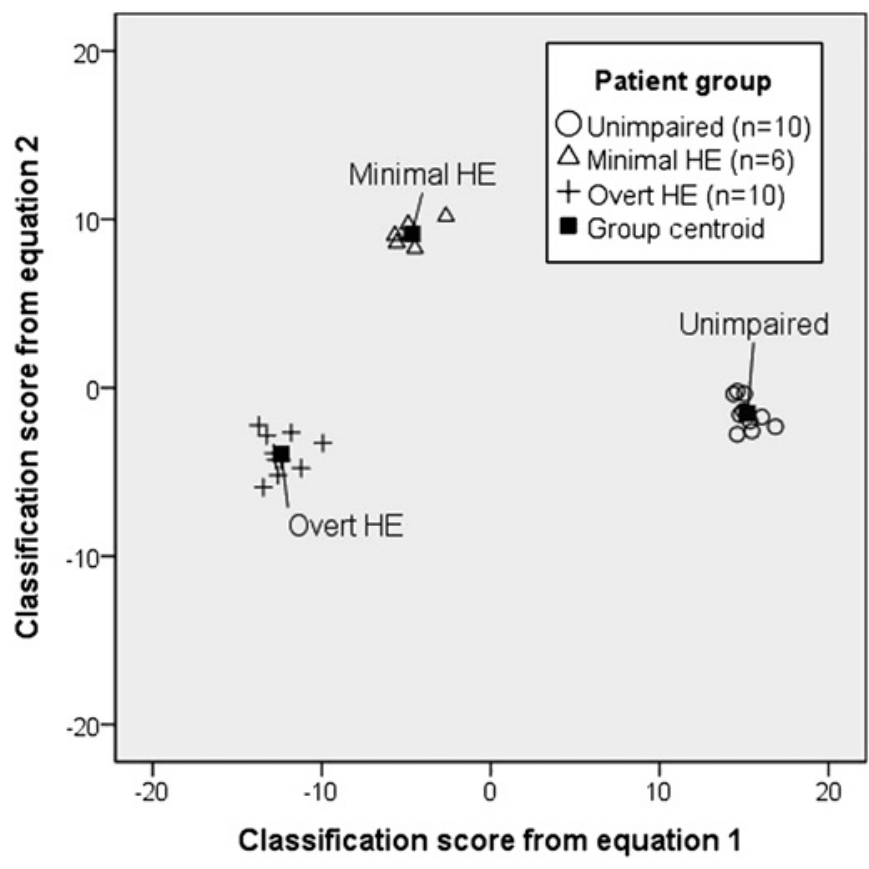

Abstract P13 Figure 1 Classification scores for the model used to predict HE. 